\title{
Técnica venográfica contrastada in vivo dos dígitos de ovinos e caprinos
}

\author{
[Contrast venography technique in vivo of the digits of sheep and goat] \\ I.F.C. Santos ${ }^{1,2}$, C.A. Hussni ${ }^{3}$, C.A. Rodrigues ${ }^{3}$, M.J. Watanabe ${ }^{3}$ A.L.G. Alves ${ }^{3}$, M. Charlier ${ }^{4}$ \\ ${ }^{1}$ Faculdade de Veterinária - Universidade Eduardo Mondlane Maputo, Moçambique \\ ${ }^{2}$ Pós-doutorando - FAPESP - FMVZ - Universidade Estadual Paulista - Botucatu, SP \\ ${ }^{3}$ Universidade Estadual Paulista - FMVZ - Botucatu, SP \\ ${ }^{4}$ Aluno de pós-graduação - FMVZ - Universidade Estadual Paulista - Botucatu, SP
}

\section{RESUMO}

Foram utilizados 10 membros torácicos e pélvicos de ovinos e caprinos hígidos, de raça e sexo variados, com idade entre dois e quatro anos e peso corpóreo médio de $53 \mathrm{~kg}$. A porção distal dos membros torácicos e pélvicos foi submetida à técnica venográfica contrastada e à contagem vascular venosa da mesma região, em ambas as espécies. Não foram observadas diferenças numéricas em relação às veias entre machos e fêmeas, nem entre os membros direito e esquerdo da mesma espécie. Os ovinos possuem maior número de veias em relação aos caprinos. A técnica venográfica distal anterógrada de ambos os membros em ovinos e caprinos se mostrou aplicável revelando a vasculatura distal e podal, a comunicação entre os vasos venosos e a quantidade dos vasos presentes na região.

Palavras-chave: casco, laminite, pequenos ruminantes, venograma

\begin{abstract}
Ten forelimbs and hindlimbs of healthy sheep and goats, of varied breeds and gender with ages ranging between two and four years and an average body weight of 53kg were used in the study. The forelimbs and hindlimbs underwent a contrasted venography of the distal region. No numerical differences were observed in relation to veins between males and females and between the left and right members of the same species. Sheep had more veins than goats. The antiretrograde venography technique of both limbs in sheep and goats was proved to be applicable, showing the vasculazation of the distal region of the foot, the communication between the vessels and the quantity of vessels.
\end{abstract}

Keywords: hoof, laminitis, small ruminants, venogram

\section{INTRODUÇÃO}

A venografia é utilizada para o diagnóstico de afecções podais em equinos e ruminantes, fornecendo maior precisão no tratamento, monitoramento e no prognóstico da doença podal, por meio da realização dos venogramas seriados (Rucker et al., 2006; Lyle, 2007; Hunt e Wharton, 2010; Rucker, 2010). A angiografia tem sido bastante utilizada na pesquisa de afecções podais com maior porcentagem em equinos, diferentemente de bovinos e pequenos ruminantes (Burns e Cornell, 1981). Visto que a anatomia vascular dos dígitos dos pequenos ruminantes é similar à dos bovinos, os mesmos podem servir de modelo para o estudo das afecções podais em bovinos (Burns e Cornell, 1981).

Segundo Rucker (2010), a padronização da técnica de venografia contrastada e a sua realização em animais hígidos é importante para o reconhecimento do padrão de contraste normal da região podal e, não menos importante, os artefatos que possam surgir durante $o$ exame venográfico. Concomitantemente, a padronização da técnica auxilia na prática clínica e/ou cirúrgica (Rucker, 2010).

Recebido em 2 de setembro de 2014

Aceito em 7 de julho de 2015

E-mail: ivansantos7@hotmail.com 
Devido ao elevado número de afecções podais que os ruminantes estão sujeitos e à falta de informação e acesso aos cuidados preventivos profissionais, associados à escassa literatura sobre a técnica venográfica da região podal em pequenos ruminantes, o trabalho tem como objetivo estudar a técnica venográfica contrastada in vivo dos dígitos dos membros torácicos e pélvicos dos ovinos e caprinos, e determinar a quantidade de veias presentes região.

\section{MATERIAL E MÉTODOS}

Foram utilizados 10 membros torácicos e pélvicos, esquerdo e direito, de ovinos e caprinos hígidos, de raça e sexo variados, com idade entre dois a quatro anos e peso corpóreo médio de 53 $\mathrm{kg}$ (42 a $63 \mathrm{~kg}$ ). Os animais foram previamente examinados quanto à sua higidez, confirmada pelo exame físico, exame clínico do casco, ausência de histórico de claudicação e exames laboratoriais - eritrograma, leucograma e avaliação plaquetária; exame sérico bioquímico (ureia, creatinina, fosfatase alcalina, aspartato aminotransferase e gama glutamil transferase). Os animais inclusos no estudo foram previamente submetidos ao exame coprológico e vermifugados de acordo com o resultado do exame. Os animais foram alocados em baias de 5 $\mathrm{X} 4$ metros, em grupos de cinco animais por espécie, e a alimentação foi à base de feno e alfafa fresca, e água ad libitum.

Antes da realização dos exames venográficos, os animais foram submetidos a jejum alimentar de 8 horas. Os cascos foram limpos com água e sabão neutro, dando especial atenção à região entre os dígitos. Paralelamente, foi realizado o casqueamento em todos os animais. Os vestígios de antisséptico iodado nos cascos foram removidos por meio de raspagem.
As faces medial e lateral das regiões metacárpicas e metatársicas foram submetidas à tricotomia, e a pele foi preparada assepticamente com álcool idodado 0,1\% (Álcool Iodado 0,1\%, Rioquimica, Campinas, São Paulo, Brasil). Em seguida, os animais foram tranquilizados com diazepam (Labesfal $^{\circledR}$, Labesfal, São Paulo, Brasil) na dose de $0,3 \mathrm{mg} / \mathrm{kg}$, por via intravenosa jugular, e contidos fisicamente em decúbito lateral, com auxílio de laçadas dos membros, excetuando o membro a ser submetido ao exame venográfico.

Foi realizado um torniquete com tubo de borracha no terço proximal do metacarpo/ metatarso, logo abaixo do carpo/tarso, e este fixado com auxílio de uma pinça hemostática tipo Kelly. O torniquete foi aplicado tendo o cuidado de se evitar alteração da posição anatômica dos vasos da região. As venopunções foram realizadas com escalpe $21 \mathrm{G}$ (Dispositivo para infusão intravenosa, Embramac ${ }^{\mathbb{B}}$, São Paulo, Brasil) em sentido anterógrado. Nos membros torácicos, de ambas as espécies, foi acessado o ramo profundo da veia radial (Popesko, 1978; Schummer et al., 1981) - acesso medial da região metacárpica. Nos membros pélvicos dos ovinos, foi acessada a veia dorsal pedis, enquanto nos caprinos foi acessado o ramo profundo da veia metatársica dorsal pedis III. Ambos os acessos foram na região lateral metatársica (Popesko, 1978; Schummer et al., 1981; Ghoshal, 2008) (Fig. 1A e 1B).

Após o preenchimento de sangue no tubo extensor do escalpe, seringa de $10 \mathrm{~mL}$ contendo diatrizoato de meglumina 60\% (Reliev $^{\circledR}$ - Justesa Imagem do Brasil SA, São Paulo, Brasil) foi acoplada no escalpe, e o meio de contraste foi aplicado lentamente e em velocidade constante (Fig. 2A, 2B e 3) 

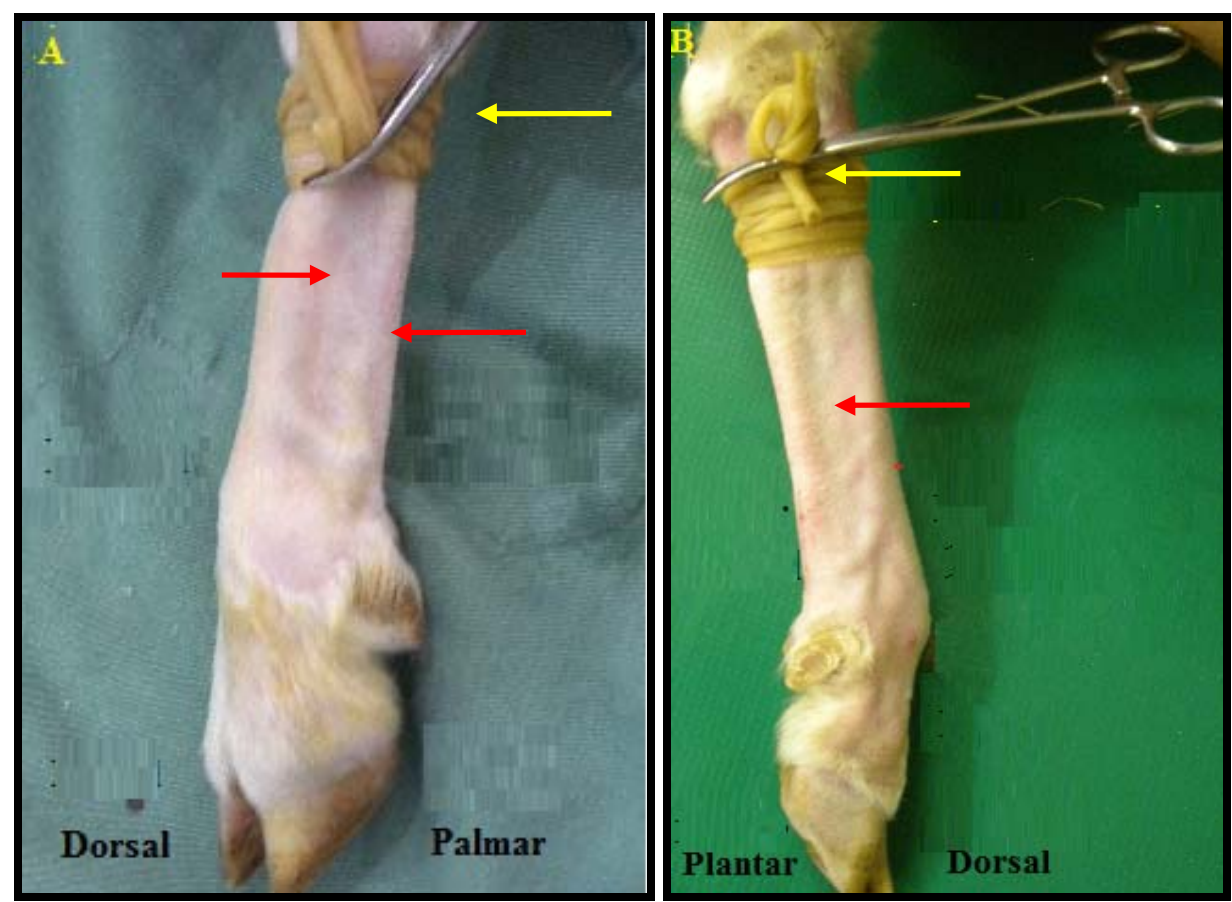

Figura 1. A - Torniquete com tubo de borracha, fixado no terço proximal do metacarpo (seta amarela) e identificação do ramo profundo da veia radial (seta vermelha) - acesso medial, membro torácico direito de ovino. B - Torniquete com tubo de borracha, fixado no terço proximal do metatarso (seta amarela) e identificação da veia metatársica dorsal pedis III (seta vermelha) - acesso lateral, membro pélvico esquerdo de ovino (Arquivo pessoal, 2014).
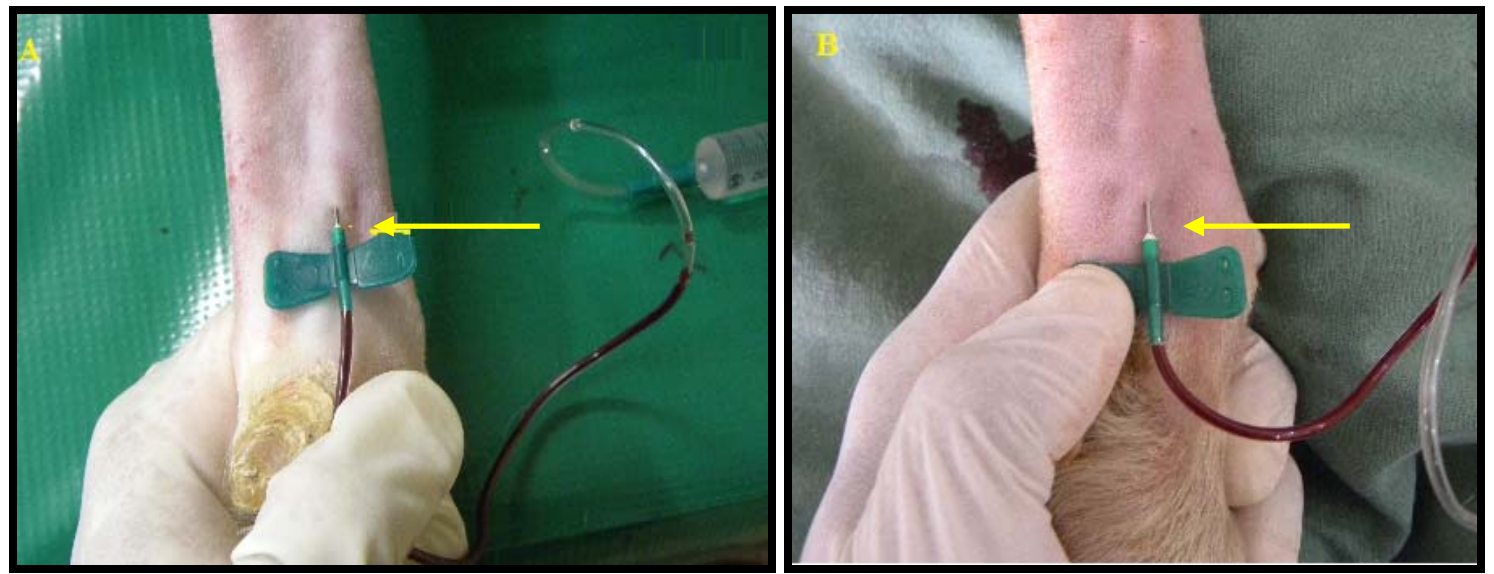

Figura 2. A - Venopunção do ramo profundo da veia radial. B - Venopunção da veia dorsal pedis. Escalpe $21 \mathrm{G}$ no sentido anterógrado (seta amarela), no membro torácico (A) e pélvico direito (B) de ovino (Arquivo pessoal, 2014).

Posteriormente à administração do meio de contraste, o escalpe foi retirado e realizou-se uma pressão digital no local da venopunção durante 20 segundos. Trinta segundos após a administração do contraste foram realizados os venogramas, utilizando o aparelho de raio-X digital (Vatech ${ }^{\circledR}$, Gnatus, Ribeirão Preto, São Paulo, Brasil) (100kVp e 45mAs). Os padrões de exposição utilizados foram de $60 \mathrm{kVp}$ e 5 mAs, com distância foco-filme (DFF) de $70 \mathrm{~cm}$, nas projeções mediolateral, dorsopalmar/ dorsoplantar e palmarodorsal/plantodorsal. 


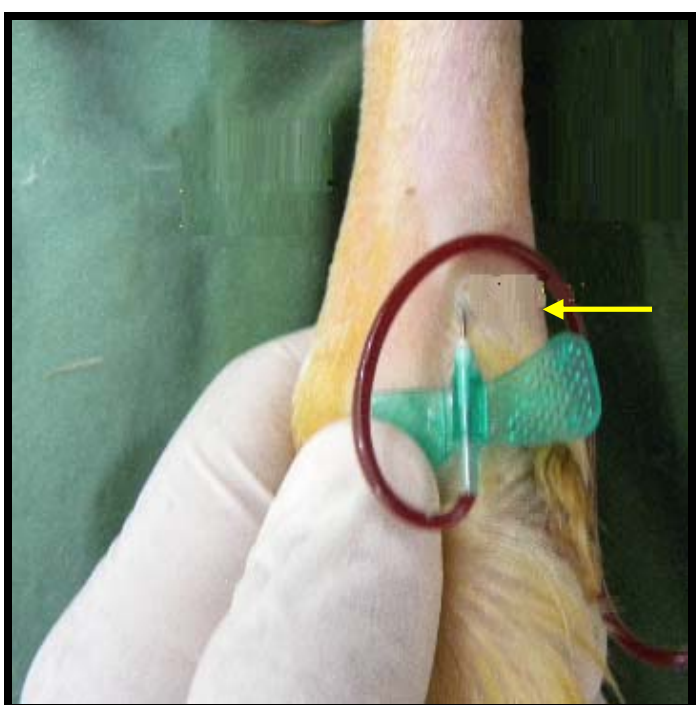

Figura 3. Venopunção do ramo profundo da veia radial, membro torácico direito de caprino, utilizando o escalpe $21 \mathrm{G}$ no sentido anterógrado (seta amarela) (Arquivo pessoal, 2014).

Durante a realização das venografias, o feixe de raio-X foi perpendicular ao chassi em todas as projeções, e o casco permaneceu centralizado no meio do chassi. Na projeção mediolateral, o feixe foi focalizado acima da superfície de apoio do casco, sendo horizontal e perpendicular em relação ao eixo longitudinal do casco.

O tempo de abrangência do meio de contraste à porção distal dos membros torácicos e pélvicos, em ambas as espécies, e o tempo desde a aplicação do meio de contraste até a difusão completa do mesmo foram cronometrados utilizando-se um cronômetro digital de precisão (Martin LCD Volume Rate ${ }^{\circledR}$, New York, USA). Os respectivos tempos foram determinados através dos venogramas seriados com intervalo de 10 segundos durante 60 segundos.

Após a realização dos exames venográficos, o torniquete foi removido e os venogramas considerados de boa qualidade radiográfica foram utilizados no estudo. Para a determinação dos venogramas de boa qualidade radiográfica, foi observado o preenchimento total das veias pelo meio de contraste de ambos os membros da região do metacarpo/metatarso, falange proximal, falange média e distal. Os respectivos venogramas foram submetidos à avaliação cega, por dois avaliadores, utilizando-se o programa EcoView ${ }^{\circledR}$, sendo avaliadas a difusão e a abrangência das estruturas pelo contraste nos sentidos distal, palmar/plantar, dorsal e contralateral. As contagens dos vasos nas regiões supracitadas foram tomadas três vezes em cada membro e posteriormente submetidas a uma média aritmética e desvio padrão.

O estudo foi realizado de acordo com os princípios éticos do Colégio Brasileiro de Experimentação Animal e aprovado pela Comissão de Ética, com protocolo número 65/2011 - CEUA.

\section{RESULTADOS E DISCUSSÃO}

A técnica de venografia in vivo da porção distal ao carpo e tarso, incluindo os dígitos, dos ovinos e caprinos é escassa na literatura, diferentemente em equinos e bovinos (Boosman et al., 1989; Singh et al., 1994; Rebhun e Pearson, 1995; Redden, 2001a; Rucker et al., 2006; Lyle, 2007; Brunner et al., 2008; Redden, 2009; Rucker, 2010; Hunt e Wharton, 2010; Baldwin e Pollitt, 2010; D’Arpe e Bernardini, 2010; Rafael et al., 2011). Segundo D’Arpe e Bernardini (2010), a técnica em animais hígidos permite estudos quanto à vascularização em processos mórbidos.

De acordo com Nazhvani et al. (2007), o efeito de sobreposição vascular durante a realização do exame venográfico impossibilita a determinação do número exato dos vasos, o mesmo foi observado no presente estudo. Visto ser subjetiva a determinação da quantidade de veias por meio do venograma, a metodologia utilizada no presente estudo possui valor na clínica veterinária de pequenos ruminantes.

Os debris e resquícios do antisséptico iodado localizados no casco dos animais foram removidos com o intuito de se evitar ou diminuir a incidência de artefatos durante o exame venográfico.

A posição do torniquete na região do terço proximal do metacarpo/metatarso foi eficaz para ocorrer o ingurgitamento das veias, sendo coerente com a literatura (Boosman et al. 1989).

A venopunção realizada em sentido anterógrado foi determinante para a realização dos exames venográficos com os animais em decúbito lateral, possivelmente devido à posição do operador em relação ao membro do animal, como também a 
não ativação das válvulas intraluminais e menor diferença de hemodinâmica causada (Meissner et al., 2007; Franceschi e Zamboni, 2009; Santos, 2014). Por outro lado, em estudo realizado por Rebhun e Pearson (1995), foi observado que, em animais em posição quadrupedal, a colocação e a manutenção do escalpe no sentido retrógrado foi essencial para realização dos exames venográficos. Os vasos de venopunção foram de acordo com Popesko (1978), Schummer et al. (1981), Ramos et al. (2012) e Santos (2014). O vaso de venopunção do membro torácico, ramo profundo da veia radial, em ambas as espécies, foram observados mais finos, menos tortuosos e medialmente em relação aos vasos do membro pélvico dos ovinos e caprinos, veia dorsal pedis (ovinos) e ramo profundo da veia metatársica dorsal pedis III (caprinos). O volume de $10 \mathrm{~mL}$ de meio de contraste utilizado em cada membro para realização dos venogramas foi suficiente para abranger a região distal dos membros, incluindo os dígitos, coerente com Ramos et al. (2012) e Santos (2014). Paralelamente, a velocidade de administração do meio de contraste foi lenta e constante, objetivando-se evitar a ruptura vascular e o aumento brusco da diferença de pressão intravascular. Desse modo, proporcionou-se uma vasodilatação gradual e, consequentemente, maior quantidade de contraste na porção distal dos membros (Lyle, 2007; Brunner et al., 2008; Redden, 2009).

O tempo que o meio de contraste levou para alcançar os dígitos dos membros torácicos $\mathrm{e}$ pélvicos, em ambas as espécies, foi de 30 segundos, o mesmo observado por Ramos et al. (2012) e Santos (2014) em ovinos e caprinos. Por outro lado, o tempo desde a administração do meio de contraste até a difusão completa do mesmo foi de 60 segundos, estando de acordo com Brunner et al. (2008) em bovinos, e Ramos et al. (2012) e Santos (2014) em ovinos e caprinos.

Entre a projeção mediolateral, dorsopalmar e palmarodorsal, e a dorsoplantar e plantodorsal foram observadas diferenças no número de vasos em ovinos (Fig. 4A, 4B e 4C) e caprinos (Fig. $5 \mathrm{~A}, 5 \mathrm{~B}$ e $5 \mathrm{C}$ ), demonstrando a necessidade de realização de todas as projeções, similar ao citado por Ramos et al. (2012) e Santos (2014), e divergente de McEvoy et al. (2002) e Nazhvani et al. (2007), citando apenas duas projeções, lateromedial e dorsopalmar e/ou dorsoplantar.
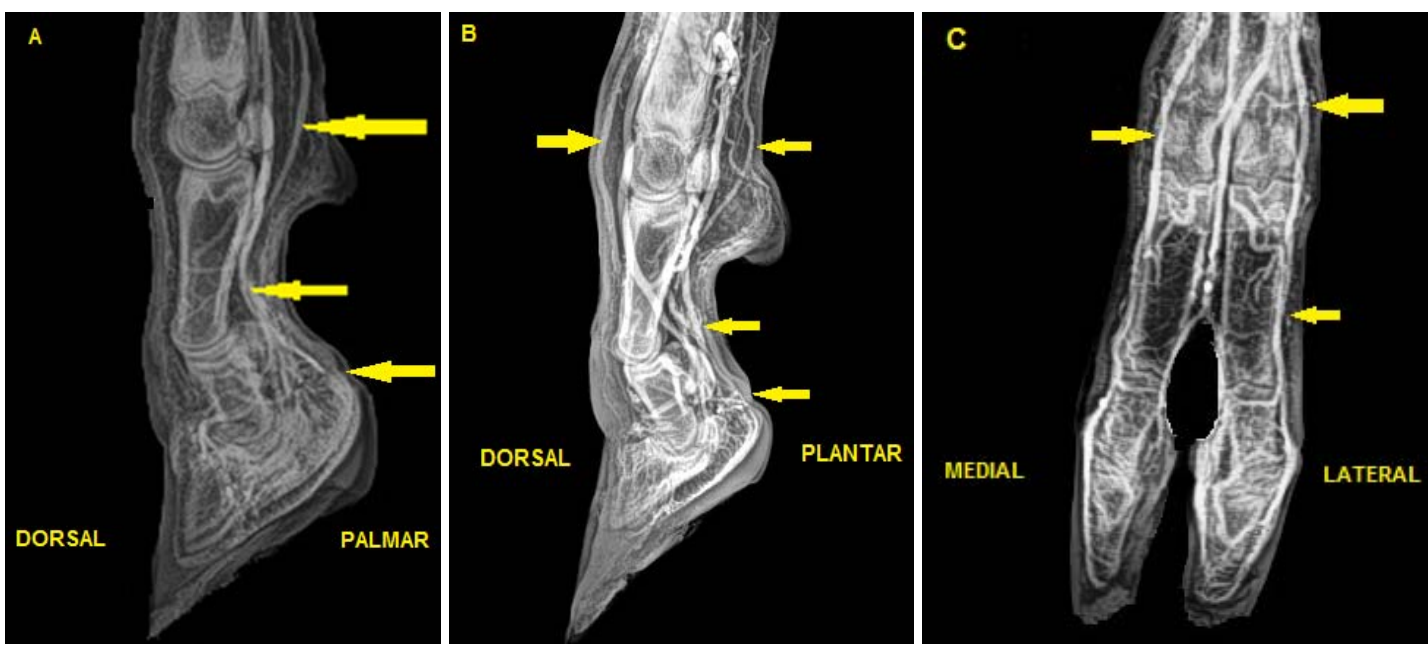

Figura 4. Venogramas da região distal do metacarpo, falanges proximal, média e distal de ovino, ilustrando as veias das regiões (setas amarelas). A - Projeção lateromedial do membro torácico; B - Projeção lateromedial do membro pélvico; C - Projeção palmarodorsal do membro pélvico $(60 \mathrm{kVp}$; 5mAs, DFF: 70cm) (Arquivo pessoal, 2014). 

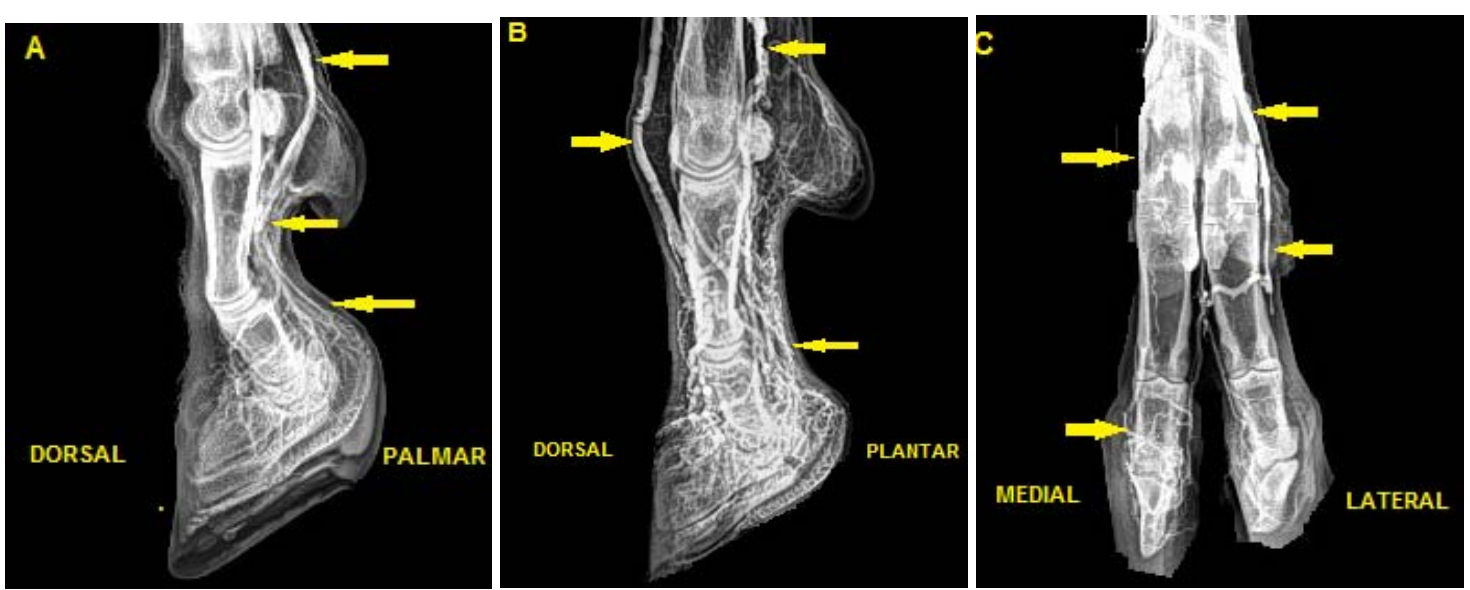

Figura 5. Venogramas da região distal do metacarpo, falanges proximal, média e distal de caprino, ilustrando as veias das regiões (setas amarelas). A - Projeção lateromedial do membro torácico; B Projeção lateromedial do membro pélvico; C - Projeção palmarodorsal do membro pélvico $(60 \mathrm{kVp}$; 5mAs, DFF: 70cm) (Arquivo pessoal, 2014).

As Tabelas 1 e 2 resumem a contagem das veias distais ao metacarpo/metatarso, e das regiões das falanges proximal, medial e distal do membro torácico e pélvico dos ovinos e caprinos, nas projeções mediolateral, dorsopalmar, dorsoplantar, palmarodorsal e plantodorsal. Para tal, foram incluídas na contagem as veias de maior calibre observadas nas regiões acima citadas.

Tabela 1. Número de veias (média) das regiões distais ao metacarpo (MTC), metatarso (MTT), falanges proximais (FP), falanges médias (FM), falanges distais (FD) dos membros torácico (MT) e pélvico (MP) de ovinos, nas projeções mediolateral (PML), dorsopalmar (PDPa), dorsoplantar (PDPl), palmarodorsal (PPaD) e plantodorsal (PPID).

\begin{tabular}{lcclll}
\hline Projeções & Membros & MTC/MTT & FP & FM & FD \\
\hline \multirow{2}{*}{ PML } & MT & 2 & 2 & 2 & 1 \\
& MP & 4 & 4 & 2 & 1 \\
\hline PDPa & MT & 3 & 3 & 4 & 4 \\
PDPl & MP & 2 & 3 & 4 & 4 \\
PPaD & MT & 3 & 3 & 4 & 2 \\
PPID & MP & 4 & 3 & 4 & 4 \\
\hline
\end{tabular}

Botucatu. São Paulo. 2014.

Tabela 2. Número de veias (média) das regiões distais ao metacarpo (MTC), metatarso (MTT), falanges proximais (FP), falanges médias (FM), falanges distais (FD) dos membros torácico (MT) e pélvico (MP) de caprinos, nas projeções mediolateral (PML), dorsopalmar (PDPa), dorsoplantar (PDPl), palmarodorsal (PPaD) e plantodorsal (PPID).

\begin{tabular}{llllll}
\hline Projeções & Membros & MTC/MTT & FP & FM & FD \\
\hline \multirow{2}{*}{ PLM } & MT & 2 & 2 & 1 & 1 \\
& MP & 2 & 2 & 1 & 1 \\
\hline PDPa & MT & 2 & 5 & 3 & 3 \\
PDPl & MP & 3 & 5 & 4 & 4 \\
PPaD & MT & 3 & 6 & 4 & 2 \\
PPID & MP & 3 & 5 & 4 & 3 \\
\hline
\end{tabular}

Botucatu. São Paulo. 2014. 
Com a metodologia utilizada no presente estudo, não foram observadas diferenças numéricas em relação às veias entre machos e fêmeas, nem entre os membros direito e esquerdo da mesma espécie. Por outro lado, foi possível identificar maior número de veias nos ovinos em relação aos caprinos. Contudo, o membro pélvico de ambas as espécies possuem maior número de veias em relação ao membro torácico.
O erro de técnica observado durante a realização dos exames venográficos em ambos os membros, de ovinos e caprinos, foi o extravasamento do meio de contraste para o espaço perivascular, totalizando $20 \%$ e $15 \%$ nos membros torácicos de ovinos e caprinos, respectivamente; enquanto nos membros pélvicos foi de $15 \%$ nos ovinos e $10 \%$ nos caprinos (Fig. 6A e 6B).
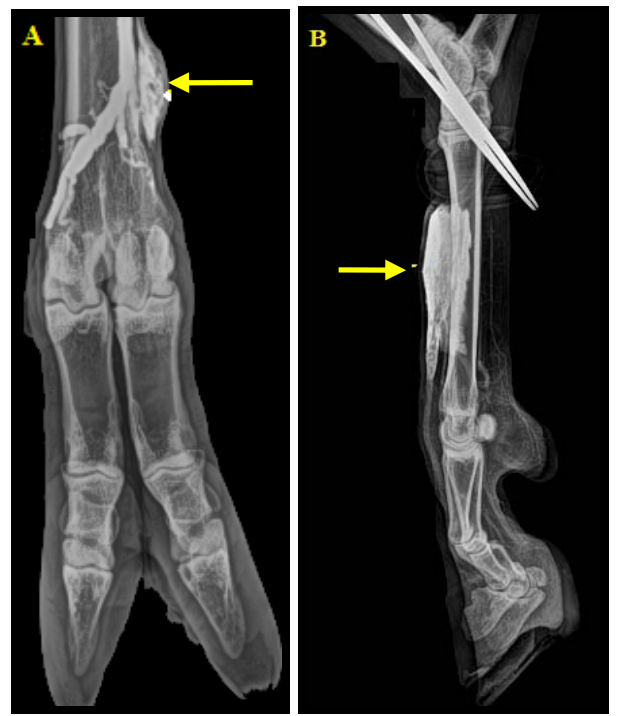

Figura 6. A. Venograma em projeção dorsopalmar da região distal do membro torácico esquerdo de ovino, evidenciando a perda de contraste no local da venopunção (seta amarela), resultando na ausência de preenchimento venoso na região distal. B - Venograma em projeção mediolateral da região distal do membro pélvico direito de caprino, ilustrando a presença de contraste no espaço perivascular (seta amarela) (Arquivo pessoal, 2014).

Concomitantemente, Redden (2009), McEvoy et al. (2002) e Ramos et al. (2012) consideraram o extravazamento do meio de contraste para o espaço perivascular como o erro de técnica mais comum durante a venografia em cavalos e ovinos, respectivamente.

Os erros de técnica poderiam ser evitados substituindo-se o tipo de torniquete e utilização de bloqueio anestésico. O torniquete de tubo de borracha utilizado no presente estudo gerou incômodo e consequentemente estresse em todos os animais, observado pela agitação nos mesmos após a aplicação do torniquete. Redden (2001a) e Rucker (2010) citaram o uso de torniquete pneumático ou esmarch, evitando o desconforto causado pelo trauma direto dos tecidos moles. Porém, a utilização desses tipos de torniquete em ovinos e caprinos ocuparia maior área nos membros dos mesmos em relação aos bovinos e equinos, dificultando o acesso venoso desejado. Paralelamente, durante a administração do meio de contraste, foi observada, em todos os animais, a retração dos membros. Essa retração poderá estar relacionada com a sensação de calor e ardência devido ao $\mathrm{pH}$ ácido do meio de contraste, e distensão venosa que ocorre durante a administração do meio de contraste. Segundo Brunner et al. (2008), a retração resultou na formação de hematoma e venogramas de baixa qualidade radiográfica em bovinos. Essa retração poderia ser evitada com bloqueio anestésico, porém, incrementando o custo do exame. Em equinos, o extravasamento do meio de contraste esteve relacionado com o tipo de contenção, $\mathrm{pH}$ ácido do meio de contraste e distensão venosa (Brunner et al., 2008; Hunt e Wharton, 2010; Baldwin e Pollitt, 2010; A'Arpe e Bernardini, 2010), o mesmo observado no presente estudo. 
Durante o estudo, não foi observada formação de hematomas nem irritação tecidual localizada devido ao extravasamento do meio de contraste, contrariamente ao citado por Nieto (2005), referenciando a presença de eritema no local do extravazamento após 48 horas. Concomitantemente, não foram observadas reações adversas com relação ao meio de contraste, porém, sendo raras, podem ser observadas em 5\% dos animais submetidos ao uso do diatrizoato de meglumina (Wannmacher, 2008). De acordo com Nieto (2005) e Wannmacher (2008), podem ocorrer reações adversas mínimas devido ao uso do meio de contraste, como êmese, tonturas, arritmia transitória e edema facial.

\section{CONCLUSÕES}

A venografia distal anterógrada do membro torácico e pélvico de ovino e caprino se mostra aplicável, revelando a vasculatura distal e podal, e a comunicação entre os vasos venosos com abrangência em todas as direções do membro. De acordo com a metodologia utilizada, os ovinos possuem maior número de veias em relação os caprinos, e em ambas as espécies são observadas maior quantidade de veias no membro pélvico em relação ao membro torácico, podendo variar de acordo com a metodologia utilizada devido à sobreposição de vasos. O erro de técnica venográfica que se observa é o extravasamento do meio de contraste para o espaço perivascular.

\section{AGRADECIMENTOS}

À CAPES (Coordenação de Aperfeiçoamento de Pessoal de Nível Superior), pela bolsa de doutorado de Ivan Felismino Charas dos Santos. À Juliana Alonso e Giovanna Brombini, pelo apoio na realização dos exames venográficos.

\section{REFERÊNCIAS}

BALDWIN, G.I; POLLITT, C.C. Progression of venographic changes after experimentally induced laminitis. Vet. Clin. North. Am. Equine Pract., v.26, p.135-140, 2010.

BOOSMAN, R.; NEMETH, F.; GRUYS, E. et al. Arteriographical and pathological changes in chronic laminitis in dairy cattle. Vet. Quart. v.11, p.144-155, 1989.
BRUNNER, C.H.M.; MARTINS, M.F.M.; BOVINO, E.E. Angiografia in vivo para avaliação da vascularização do casco de equinos. Cienc. Rur., v.38, p.116-123, 2008.

BURNS, J.; CORNELL, C. Angiography of the caprine digit. Vet Rad and Ultrasound, v. 22, p.17-176, 1981.

D'ARPE, L.; BERNARDINI, D. Digital venography in horses and its clinical application in Europe. Vet. Clin. North Am. Equine Pract., v.26, p.339-359, 2010.

FRANCESCHI, C.; ZAMBONI, P. Venous hemodynamics. In: FRANCESCHI, C.; ZAMBONI, P. (Eds.). Principles of venous hemodynamics. Pennsylvania: Nova Science Publishers, 2009. p.38-46.

GHOSHAL, N.G. Coração e artérias. In: GETTY, R. (Ed.). Anatomia dos animais domésticos. 5.ed. Rio de Janeiro: Guanabara Koogan, 2008. p.900-960.

HUNT, R.J.; WHARTON, R.E. Clinical presentation, diagnosis, and prognosis of chronic laminitis in North America. Vet. Clin. North Am. Equine Pract., v.26, p.141-154, 2010.

LYLE, B.E. Venography as a tool for guiding surgery to the foot. In: FLOYD, A.D.; MANSMANN, R.A. (Eds.). Equine podiatry. St Louis (MO): Saunders, 2007. p.284-293.

McEVOY, F.J.; WEBBON, P.M.; GAFFNEY, P.J. An experimental clot model in sheep; generation of a heterologous clot and its detection in vivo using venography and 125I labelled fibrinogen. Res. Vet. Sci., v.72, p.217221, 2002.

MEISSNER, M. H.; MONETA, G.; BURNAND, $\mathrm{K}$. et al. The hemodynamics and diagnosis of venous disease. J. Vasc. Surg., v. 46, p. 232-244, 2007.

NAZHVANI, S.D.; ABBASI, S.; TADJALLI, M. Arteriographical evaluation of normal digit and hoof in goat. Iran J. Vet. Surg., v.2, p.43-48, 2007.

NIETO, J. Como realizar un venograma digital para evaluar caballos con laminitis. Rev Méd Equina, v.12, p.1-15, 2005.

POPESKO, P. (Ed.). Atlas of topographical anatomy of the domestic animals. Philadelphia: W.B. Saunders, 1978. Vol.3, p. 84-99. 
RAFAEL, L.A.; PYLES, M.D.; RODRIGUES, M, RODRIGUES, C.A. Venograma digital para avaliação de laminite crônica em um bovino. In: VII AMOSTRA CIENTÍFICA DE CIÊNCIAS AGRÁRIAS, XV AMOSTRA CIENTÍFICA DA FMVZ, XVIII REUNIÃO CIENTÍFICA DA FAZENDO DO LAGEADO, 2011, Botucatu. Anais...Botucatu: [3] 2011, p.23. (Resumo).

RAMOS, C.M.; HUSSNI, C.A.; SANTOS, I.F.C. Estudo anatômico venográfico da porção distal dos membros de ovinos. In: XXIV CONGRESSO DE INICIAÇÃO CIENTÍFICA DA UNESP, 24., 2012, Águas de Lindóia, São Paulo. Anais...São Paulo: UNESP, 2012, p.3-4. (Resumo).

REBHUN, W.C.; PEARSON, E.G. Clinical management of bovine foot problems. J. Am. Vet. Med. Assoc., v.180, p.1464-1467, 1995.

REDDEN, R.F. A technique for performing digital venography in the standing horse. Equine Vet. Educ., v.3, p.172-178, 2001a.

REDDEN, R.F. Possible therapeutic value of digital venography in two laminitic horses. Equine Vet. Educ., v.13, p.128-134, 2001 b.

REDDEN, R.F. Using venograms in laminitic cases. In: DR. REDDEN'S IN-DEPTH PODIATRY SYMPOSIUM, 2009, Versailles (KY). Anais ... Versailles: [2] 2009, p.81-83. (Resumo).
RUCKER, A.; REDDEN, R.F.; ARTHUR, E.G. How to perform the digital venogram. Am. Assoc. Equine Pract., v.52, p.526-530, 2006.

RUCKER, A. Clinical applications of digital venography. J. Equine Vet. Sci., v.30, p.491-503, 2010.

SANTOS, I.F.C. Venografia da porção distal dos membros de ovinos e caprinos. 2014. 108f. Tese (Doutorado em Biotecnologia Animal) Faculdade de Medicina Veterinária e Zootecnia, Universidade Estadual Paulista "Júlio de Mesquita Filho”, Botucatu, São Paulo, SP.

SCHUMMER, A.; WILKENS, H.; VOLLMERHAUS, B. et al. The circulatory system. In: NICKEL, R.; SCHUMMER, A.; SEIFERLE, E. (Eds.). The anatomy of domestic animals. Berlin: Springer - Verlag, 1981. Vol.3, p.211-227.

SINGH, S.S.; WARD, W.R.; MURRAY, R.D. An angiographic evaluation of vascular changes in sole lesions in the hooves of cattle. Br. Vet. J., v.150, p. 41, 1994.

WANNMACHER, L. (Ed.). Textos básicos de saúde. Brasília: Ministério da Saúde, 2008. p.81-85. 